\title{
Abductor reconstruction with gluteus maximus transfer in revision hemiarthroplasty
}

\author{
N Abhilash ${ }^{1, *}$, Ronald Joseph Menezes ${ }^{2}$, Jubin Abraham ${ }^{3}$ \\ ${ }^{1}$ Postgraduate Resident, ${ }^{2}$ Professor, ${ }^{3}$ Senior Resident, Dept. of Orthopaedics, Father Muller Medical College Hospital, \\ Mangalore, Karnataka, India \\ *Corresponding Author: N Abhilash \\ Email: amithabhilash@gmail.com
}

\begin{abstract}
Introduction: Abductor deficiency can be a debilitating problem following hip arthroplasty surgery and causes severe pain, limp and instability

Description of Technique: The authors had experienced abductor deficiency secondary to hemiarthroplasy following which a revision hemiarthroplasty was done and abductor reconstruction was performed using the anterior fibres of gluteus maximus. This flap served as a substitute for gluteus medius and minimus. Ultimately, both limp and pain showed prominent reduction.
\end{abstract}

Keywords: Abductor reconstruction with gluteus maximus, Revision hemiarthroplasty.

\section{Introduction}

The primary hip abductors are comprised by the tensor fascia latae, gluteus medius and minimus. All the abductors function firstly by causing hip abduction and seconding involed in balancing the body weight and also in case of non-weight bearing leg while walking. ${ }^{1}$ Theses abductors are susceptible to tear in case chronic inflammation, infection and multiple surgeries. These tears can be in the form of total or partial disruption, seen following hip revision surgery, which leads to pain and limp. This adds up to the implant instability. ${ }^{2}$ These tendon tears could be present pre-operatively or sustained as an iatrogenic complication either intraoperatively or post-operatively. There are three causes of abductor muscle ruptures as postulated by Lachiewicz. They are degenerative or traumatic tears associated with the surgical approach for arthroplasty in femoral fractures or osteoarthritis and avulsion or repair failure as a result of anterolateral or transgluteal surgical approach. ${ }^{3}$ The incidence of abductor deficiency ranges from $0.08 \%$ to $22 \%$ as documented in different studies. However, literature proves to be insufficient regarding the management and outcome of abductor deficiency. ${ }^{4}$

\section{Case Report}

An eighty year old male, presented to our hospital with a history of severe right hip pain which was sudden in onset and non radiating. Two weeks ago, he sustained a traumatic injury and was diagnosed to have a fracture of the neck of femur. He was treated in another centre where he underwent a hemiarthroplasty and an Austin Moore's prosthesis was inserted. He reported having minimal pain post operatively. He had not been mobilized post surgery and does not give any history of trauma to the right hip. On examination, the right lower limb was internally rotated and shortened. Detailed clinical examination could not be performed due to severe pain. Initially, plain radiographs of the pelvis on anteroposterior and lateral views were taken. In view of the radiographic findings, he was diagnosed with dislocation of the inserted prosthesis.

With the above diagnosis, he was taken up for revision hemiarthroplasty. After being evaluated by anesthetist, he was put in the left lateral decubitus position. The skin incision was made as accordingly done in posterolateral approach of hip. Skin incision was deepened till the gluteus maximus was exposed and it was split, as is routinely performed. On further deep dissection, the short external rotators were identified and cut. Following which, the prosthesis was found to be dislocated posteriorly and it was removed. The abductors were found to be torn and unrepairable. The trial implants were inserted in the acetabulum and femur and accordingly, AMP prosthesis of appropriate dimensions was placed. In view of history of unstable prosthesis, we planned to stabilize the same using gluteus maximus muscle fibres. So, the anterior fibres were used to reconstruct the abductors of the hip joint. In order to perform that, gluteus maximus of about 10 $\mathrm{cm}$ in length and $3 \mathrm{~cm}$ in width was harvested in the region approximately $4 \mathrm{~cm}$ distal to the tip of the greater trochanter. Te width was progressively increased at the proximal portion of harvested muscle fibres in a fan-like manner. Non-absorbable suture material was used to reconstruct. Krackow suture technique was used to suture the harvested distal portion of muscle. At the distal portion of greater trochanter, a hole of about $1 \times 1 \mathrm{~cm}$ was made so that the muscle could be passed through from medial to lateral. Following which, the suture was fixed to bone tunnel, located $3 \mathrm{~cm}$ distal to the hole in 20 degree abduction.

Post-operatively, the patient was started on the rehabilitation program. He was prevented from doing active abduction for 3 weeks. After 3 weeks, the patient was started on gradual strengthening exercise and weight bearing mobilization. 

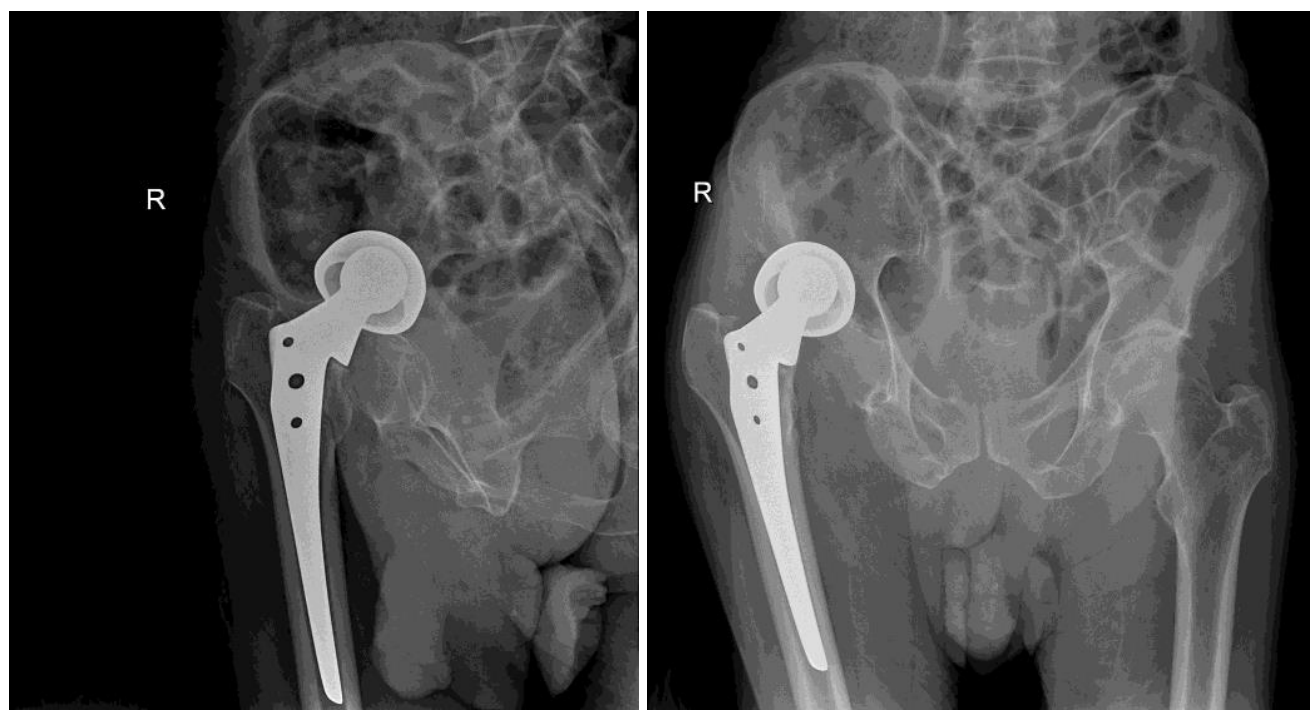

Fig. 1
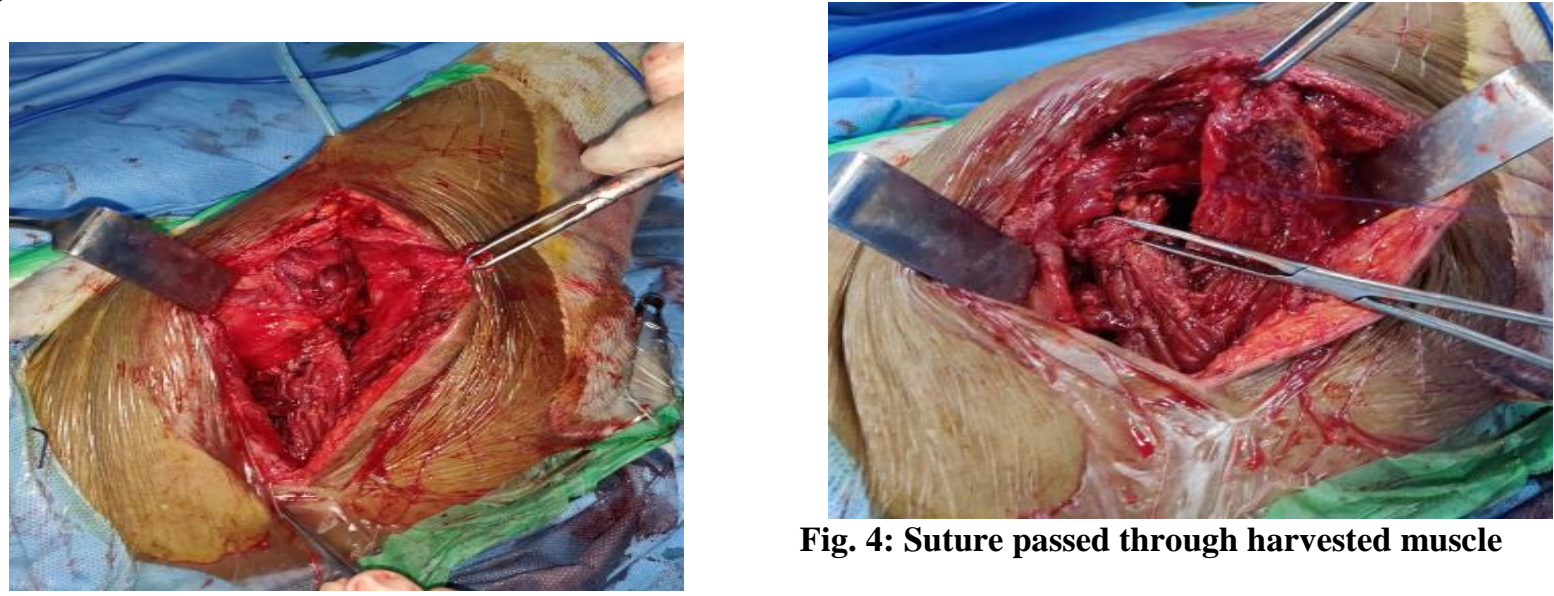

Fig. 4: Suture passed through harvested muscle

Fig. 2: Deficient gluteus medius and minimus

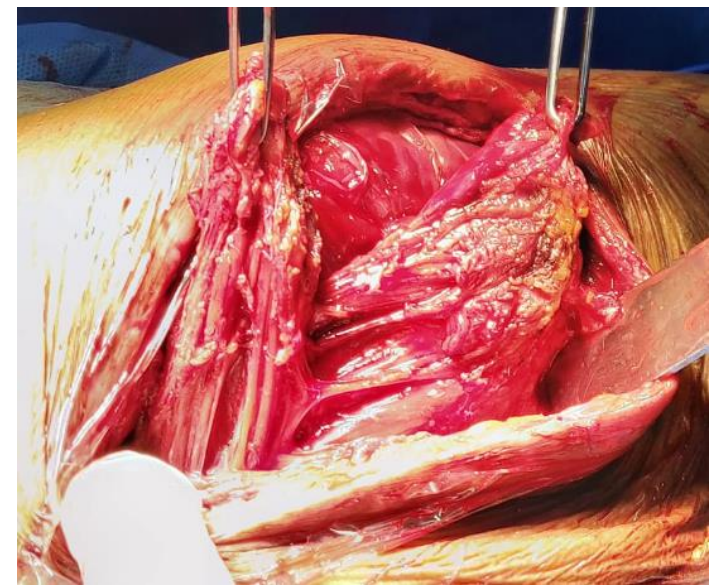

Fig. 3: Harvested gluteus maximus muscle

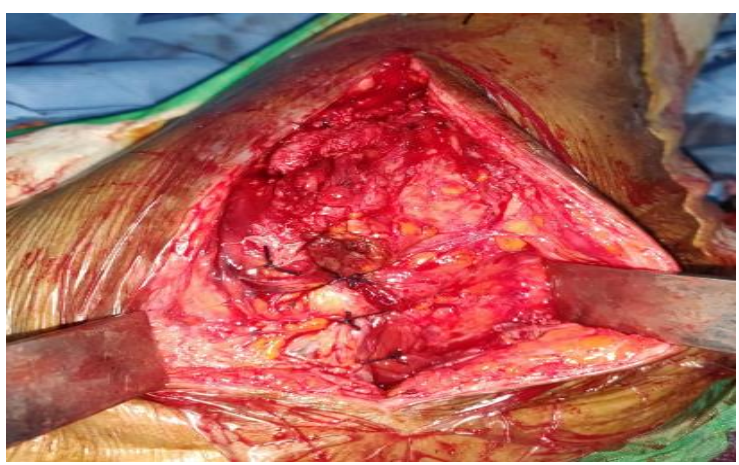

Fig. 5: Completed reconstruction

\section{Discussion}

In the native joint, deficiency of the hip abductors can come with complaints of severe hip pain and instability. On the other hand, joint instability is a more serious complication in an artificial joint. Some of the causes attributed to secondary abductor deficiency are the abductor tendon rupture, intra-operatively during total hip replacement when one fails to repair abductors, the superior gluteal nerve injury and multiple revision hip surgeries. ${ }^{1}$ In this particular case, there was a significant history of trauma to the hip as well as prior 
surgical history and either of these could have been the root cause for the deficiency of the abductor system. It has been found that if the gluteus maximus is directly attached to the greater trochanter, it can help to provide stability to the pelvis and eliminate the pelvic sag and both of these aids in eliminating the limp. ${ }^{5}$

Literature has shown many techniques which described about reconstructing the abductor mechanism. In a study by Kohl et al. the abductor defect was repaired using a vastus lateralis muscle shift and the muscle strength obtained post operatively in most of the patients was sufficient for unassisted walking with a slight limp. This indicated a good outcome. But their study was found to have a higher complication rate and required greater surgical expertise. $^{6}$

Fehm et al. in their study used an Achilles tendon allograft and calcaneal block to replace the abductor defect. Their study population composed of patients with prior history of total hip replacement and on follow up, the musculotendinous portion of the abductor muscles were found to be avulsed from the insertion site. They noted a significant improvement in the pain scale post-operatively. ${ }^{7}$

In a study on the functional anatomy of the glutei and tensor fascia latae, the major abductor was found to the tensor fascia latae. The muscle fibres of gluteus medius consists of three distinct parts. These form the fan shape. The gluteus medius and minimus show parallel fibres to the neck of femur, in their posterior aspect. The greater trochanter has insertion of these fibres distally, at the anterosuperior margin. The authors made an observation about the function of the gluteus medius and minimus primarily as hip stabilizers and pelvic rotators. With respect to this study, they concluded the anterior fibers of gluteus maximus can used to achieve joint stability because the horizontal fibres of abductors are parallel to gluteus maximus. ${ }^{8}$

There are two techniques for the reconstruction of the deficient abductor of the hip. These were introduced by Whiteside, according to whom the anterior fibers of gluteus maximus could be transferred and gluteus maximus and tensor fascia latae could be together transferred to reconstruct the abductors. The technique we used in our case, was the same as that of Whiteside's technique. We differed only with respect to distal fixation of harvested muscle. This was done so that the gluteus medius and minimus could be reconstructed at the same, and the femoral head in the acetabulum could be stabilized. In our method, the posterior capsule was reinforced with the posterior fibers of gluteus maximus. The factors to be considered to prevent the dislocation are the correct position of the implant, reconstruction of the deficient abductor and rehabilitation of the patient are the cornerstone to prevent the dislocation. ${ }^{5}$

There are several limitations to this study. Firstly, we have studied only one case. Secondly, the follow up period was relatively short and lastly, there was no comparative cohort for this present study.

Despite these limitations, we are of the opinion that the technique used in the above study would help to provide a better abductor function and enhanced joint stability, as a result the patient can have a better quality of life.

\section{References}

1. Jang SA, Cho YH, Byun YS, Gu TH. Abductor Reconstruction with Gluteus Maximus Transfer in Primary Abductor Deficiency during Total Hip Arthroplasty. 2016;28(3):178-181.

2. Alberton GM, High WA, Morrey BF (2002) Dislocation after revision total hip arthroplasty: an analysis of risk factors and treatment options. J Bone Joint Surg Am. 84, 1788-1792.

3. Lachiewicz PF. Abductor tendon tears of the hip: evaluation and management. J Am Acad Orthop Surg. 2011;19(7):385391.

4. Odak S, Ivory J. Management of abductor mechanism deficiency following total hip replacement. 2013;95(3):3437 .

5. Whiteside L. Surgical Technique: Transfer of the Anterior Portion of the Gluteus Maximus Muscle for Abductor Deficiency of the Hip. Clinical Orthopedics and Related Research®. 2011;470(2):503-510.

6. Kohl S, Evangelopoulos DS, Siebenrock KA, Beck M. Hip Abductor Defect Repair by Means of a Vastus Lateralis Muscle Shift. J Arthroplasty. [Internet]. 2012;27(4):625-9. Available from: http://dx.doi.org/10.1016/j.arth.2011.06.034

7. Fehm BMN, Huddleston JI, Burke DW, Geller JA, Malchau H. Repair of a Deficient Abductor Mechanism with Achilles Tendon Allograft After Total Hip Replacement. 2010;23052312.

8. Gottschalk F, Kourosh S, Leveau B. The functional anatomy of tensor fasciae latae and gluteus medius and minimus. 1989;179-189.

How to cite this article: Abhilash N, Menezes R. J, Abraham J. Abductor reconstruction with gluteus maximus transfer in revision hemiarthroplasty. Indian $\mathbf{J}$ Orthop Surg. 2018;4(4):411-413. 